Research papers

\title{
The fractional Boussinesq equation of groundwater flow and its applications
}

\author{
Ninghu $\mathrm{Su}^{*}$ \\ College of Science and Engineering, TropWater, and the Cairns Institute, James Cook University, Cairns, Queensland 4870, Australia
}

\section{A R T I C L E I N F O}

\section{Article history:}

Received 16 November 2016

Received in revised form 9 January 2017

Accepted 10 January 2017

Available online 13 January 2017

This manuscript was handled by $\mathrm{C}$.

Corradini, Editor-in-Chief

\section{Keywords:}

Fractional Boussinesq equation

CTRW

Symmetrical fractional derivatives

Micro-scale backwater effect

Anomalous groundwater flow

New formulae for recession curves

\begin{abstract}
A B S T R A C T
This paper presents a set of fractional Boussinesq equations (fBEs) for groundwater flow in confined and unconfined aquifers and demonstrates the application of one of the fBEs for groundwater discharges known as recession curves. The fBEs are formulated with two-term distributed fractional orders in time and symmetrical fractional derivatives (SFD) in space applicable to both confined and unconfined aquifers. The SFD in theory consists of the forward fractional derivative (FFD) and the backward fractional derivative (BFD). The FFD represents the forward movement of water along the direction of mainstream flow while the BFD accounts for the backward motion of water in the direction opposite to the mainstream flow. The backward flow at the pore level can be referred to as the micro-scale backwater effect. The analogue of the backwater effect on a micro-scale using the BFD coincides with the wandering processes based on the continuous-time random walk (CTRW) theory which results in the fractional governing equation. With the analytical solutions of the fBE for given initial and boundary conditions of the first type for a finite depth, a set of formulae for groundwater recession has been derived using approximate solutions of the fBE. The examples of the applications of the recession curves are graphically illustrated and the effects of the orders of fractional derivatives on the geometry of the flow curves examined.
\end{abstract}

(c) 2017 Elsevier B.V. All rights reserved.

\section{Introduction}

Groundwater is a vital resource for many sectors and human consumption in the society around the world. Its management, protection and assessment require quantitative knowledge of the flow in aquifers. The quantification of groundwater flow has been a major issue for hydrologists, engineers and applied mathematicians for more than a century, and the methods for its quantification are among the most important issues in groundwater hydrology.

The first step towards quantifying water flow in porous media was achieved by Darcy (1856), and complete mathematical formulation in the form of partial differential equations (PDEs) was presented and analysed by Boussinesq (1904). Numerous papers and books have been published since these important pioneering milestones, and extensive research is continuing which is evidenced by continuous publications in various forms.

One form of the Boussinesq equation (BE) of groundwater flow in an aquifer in two dimensions was given by Boussinesq (1904, p. 22) as

$\phi \frac{\partial h}{\partial t}=\frac{\partial}{\partial x}\left(K h \frac{\partial h}{\partial x}\right)+\frac{\partial}{\partial y}\left(K h \frac{\partial h}{\partial y}\right)$

\footnotetext{
* Corresponding author.

E-mail address: ninghu.su@jcu.edu.au
}

where

$h$ is the hydraulic head of the aquifer with a dimension $[L]$; $K$ is the saturated hydraulic conductivity of the aquifer, $[L / T]$; $\phi$ is either the effective porosity in unconfined aquifers or the storativity coefficient in confined aquifers, which are dimensionless (see Table 1 for details);

$x$ and $y$ are the space variables in two directions, $[L]$ and $t$ is the time, $[T]$.

Eq. (1) applies to both confined and unconfined aquifers but with a key difference being the distinction between the parameters and their definitions for the two types of aquifers, which are summarised by Bras (1990, p. 296-299) in Table 1.

According to Bear (1972), Boussinesq (1904) was the first to present an exact solution of Eq. (1) for analysing groundwater flow. For more than a century, the BE and its simplifications have formed the basis for analysing groundwater flow. In one dimension with the slope gradient of the aquifer base included, the BE is written as (Werner, 1957)

$\phi \frac{\partial h}{\partial t}=K\left[h \frac{\partial^{2} h}{\partial x^{2}}+\left(\frac{\partial h}{\partial x}\right)^{2}-\omega \frac{\partial h}{\partial x}\right]$

where $\omega$ is the gradient of the aquifer on an impervious base. Eq. (2) can be written as follows to accommodate the variability of the saturated hydraulic conductivity, 
Table 1

The specification of the parameters for unconfined and confined aquifers.

\begin{tabular}{lll}
\hline & Unconfined aquifer & Confined aquifer \\
\hline Definitions & Effective porosity & Storativity coefficient \\
$\phi$ & $\phi=\frac{n_{e}}{K h_{0}}$ & $\phi=\frac{S}{T}$ \\
Notes & $n_{e}$ is the effective porosity in; & $S$ is the storativity coefficient \\
& $h_{0}$ is the hydraulic head in the & or specific storage coefficient; \\
& saturated zone around which & $T$ is the transmissivity which \\
& Eq. (1) is linearised & $\begin{array}{l}\text { is now equal to } K b, \text { where } b \text { is } \\
\text { the thickness of the confined } \\
\end{array}$ \\
& & aquifer
\end{tabular}

$\phi \frac{\partial h}{\partial t}=\frac{\partial}{\partial x}\left[K h\left(\frac{\partial h}{\partial x}-\omega\right)\right]$

Clearly, Eq. (3) is a consequence of the conservation of mass given by

$\frac{\partial h}{\partial t}=-\frac{\partial q}{\partial x}$

where $q$ is the mass flux through a cross section, $B$, given by (Werner, 1957)

$q=K h\left(\omega-\frac{\partial h}{\partial x}\right)$

In addition to the application and continual analysis of the $\mathrm{BE}$ (Bear, 1972; Barenblatt et al., 2000; Abdellaoui et al., 2015; Telyakovskiy et al., 2016), over the past twenty years or so various forms of the fractional Boussinesq equation (fBE) have been presented based on the fractional calculus. Some of the forms are given by He (1998), Atangana and his colleagues (Atangana and Bildik, 2013; Atangana, 2014; Atangana and Vermeulen, 2014; Alkahtani and Atangana, 2016; Atangana, 2016; Atangana and Alkahtani, 2016; Atangana and Baleanu, 2016; Djida et al., 2016), Mehdinejadiani et al. (2013) and Zhuang et al. (2014) who have demonstrated the application of fractional partial differential equations (fPDEs) to groundwater problems. These developments on water flow complement the applications of fractional calculus on solute movement in groundwater by Benson (1998) and many subsequent investigators, particularly he and his colleagues such as Benson et al. (2000a, 2000b), Schumer et al. (2003a, 2003b) and others as reviewed by Berkowitz et al. (2006), Zhang et al. (2009) and Benson et al. (2013).

In this paper, we present a two-term distributed-order timespace fBE which can be simplified into different forms, and solutions following a recent satisfactory application of a similar fPDE to groundwater flow in a radial coordinate for well hydraulics (Su et al., 2015). The fBE by definition incorporates the fractional derivatives in time and two components of fractional derivatives in space. The two components of fractional derivatives in space explain forward motion of flow and the backwater effect by the backward fractional derivative.

\section{The fractional Boussinesq equation of groundwater flow and its connection with the continuous-time random walk theory}

\subsection{A brief background}

The conception of fractional calculus was initiated more than 320 years ago (Podlubny, 1999), but its development and applications do not parallel those for integer calculus. In spite of this disparity, some concepts and methods developed for fractional calculus have found significant applications such as the fractional partial differential equations (fPDE) and their association with stochastics and fluid mechanics etc. Such examples include the application of the continuous-time random walk (CTRW) theory (Montroll and Weiss, 1965) to derive the governing equations for flow and transport in porous media without resorting to the traditional method of mass conservation (Gorenflo and Mainardi, 1998a, 1998b, 2005, 2009; Zaslavsky, 2002; Uchaikin and Saenko, 2003; Gorenflo et al., 2007). A very important feature of the fPDE so derived is related to the transport exponent (Zaslavsky, 2002), which defines the pattern of flow and transport by the two parameters in the CTRW model, and is also related to the parameters which defines the fractal space-time structures (Zaslavsky, 2002, p. 507).

The CTRW is a further development from the concept of the random walk, which was first mathematically illustrated by Crofton (1865) in terms of the random flight as an alternative term, and later used by Pearson (1905). The phenomenon described by the CTRW concept is anomalous, which is a generalisation of the transport processes with the classic diffusion as a special case (Gorenflo et al., 2007). The CTRW theory for transport is understood in the framework of the classical renewal theory (Cox, 1967), and was successfully applied to model solute movement in porous media more than 50 years ago since Saffman (1959). In recent years the CTRW theory is being more widely applied to model flow and transport in different geological formations such as aquifers and soils (Berkowitz and Scher, 1995; Benson, 1998; Benson et al., 2000b; Gorenflo and Mainardi, 2001, 2005, 2009; Berkowitz et al., 2002; Dentz and Berkowitz, 2003; Schumer et al., 2003a; Berkowitz et al., 2006; Benson et al., 2013; Dentz et al., 2015).

For detailed discussions and reviews of the connection between the CTRW and the fPDEs, the reader is referred to Uchaikin and Saenko (2003), Gorenflo et al. (2007), and Gorenflo and Mainardi (2012). Here we briefly re-state this connection for the benefit of the readers in groundwater hydrology.

The CTRW provides a framework for transport phenomena which consists of a sequence of two states of independently identically distributed (iid) processes: one state stands for the iid positive waiting times denoted by $T_{1}, T_{2}, T_{3}, \ldots$, each having the same probability density function (pdf), $\phi(t), t>0$, and the second state is the sequence of the iid random jumps denoted by $X_{1}, X_{2}, X_{3}, \ldots$ in a real domain, $\mathbf{R}$, each having the same pdf $w(x), x \in \mathbf{R}$. The probability density of the particle or water parcel movement in the media is $p(x, t)$, which is represented by the series (Gorenflo et al., 2007),

$p(x, t)=\Psi(t) \delta(x)+\sum_{n=1}^{\infty} v_{n}(t) w_{n}(x)$

where

$\Psi(t)$ is the survival function given by

$\Psi(t)=\int_{t}^{\infty} \phi\left(t^{\prime}\right) d t^{\prime}$

with $v_{n}(t)$ and $w_{n}(x)$ as the repeated convolutions in time and space, respectively, given by $v_{n}(t)=\left(\Psi * \phi^{* n}\right)(t)$, and $w_{n}(x)=\left(w^{* n}\right)(t)$. With Eq. (6), it is shown (Gorenflo et al., 2007; Gorenflo and Mainardi, 2012) that the expression

$\hat{\tilde{u}}(\kappa, s)=\int_{0}^{\infty}\left[\exp \left(-t_{*}|\kappa|^{\lambda} i^{\omega \operatorname{sign} \kappa}\right)\right]\left[s^{\beta-1} \exp \left(-t_{*} s^{\beta}\right)\right] d t_{*}$

is equivalent to

$\hat{\tilde{u}}(\kappa, s)=\frac{s^{\beta-1}}{S^{\beta}+|\kappa|^{\lambda} i^{\omega \operatorname{sign} \kappa}}$

which is the Laplace-Fourier transform of the following time-space fractional partial differential equation (fPDE)

$\frac{\partial^{\beta} u(x, t)}{\partial t^{\beta}}=\frac{\partial^{\lambda} u(x, t)}{\partial x^{\lambda}}, \quad u(x, 0)=\delta(x)$ 
where

$\kappa$ and $s$ are the Fourier and Laplace transform variables, respectively;

$\beta$ is the order of the temporal fractional derivative and

$\lambda$ the order of the spatial fractional derivative.

The above fact implies that the CTRW model in the LaplaceFourier domain is equivalent to the fPDE in the time domain, thus establishing the connection between the CTRW and the FPDE in two disciplines. The CTRW model is characterised by the longtime limit of a CTRW with power-law jumps, $P(X>x) \approx x^{-\beta}$ and power-law waiting times, $P(J>t) \approx t^{-\lambda}$ (Meerschaert, 2012) with $P(X>x)$ and $P(J>t)$ as the conditional probabilities for jumps and waiting times, respectively. Equation (10) can also be written as

${ }_{t} D_{*}^{\beta} u(x, t)={ }_{x} D_{\varpi}^{\lambda} u(x, t), \quad u(x, 0)=\delta(x)$

where the left hand side of Eq. (11) is the Caputo fractional derivative with respect to time while the right hand side is the Riesz-Feller potential (which defines the fractional derivatives) with respect to space, and $\varpi$ is the skewness. It should be noted that the RieszFeller fractional derivative becomes the Liouville fractional derivative for $\varpi= \pm \lambda$ with the positive sign for the forward fractional derivative (FFD) and the negative sign for the backward fractional derivative (BFD) (Ortigueira and Trujillo, 2012, p. 5155-5156). In the following section and Appendix A the FFD and BFD are detailed.

The above brief review shows the direct link between the CTRW model and the fPDE for the same transport process. Water flow in unsaturated soils is successfully described using the CTRW theory (Su, 2014), and the flow in saturated media should have similar properties with the saturation in the media as a special case. Given that solute movement in porous media has been successfully modelled using the CTRW theory (Meerschaert et al., 2002; Meerschaert, 2012), water flow that entrains solute movement in the media can be consistently interpreted using the CTRW concept. It is this fundamental connection and earlier successful analysis which prompt the author to extend the approach using the CTRW theory to saturated flow in aquifers which leads to the fBE.

\subsection{The fractional Boussinesq equations and their classifications}

The conventional flux or discharge, $q$, per unit width, has been modified in the literature to accommodate the fractional concept (He, 1998; Voller, 2011). Instead of a fractional gradient, here we use the fractional derivation of the flux, which can be regarded as the compact form given by Wheatcraft and Meerschaert (2008)

$\phi \frac{\partial h}{\partial t}=-\frac{\partial^{\eta} q}{\partial x^{\eta}}, \quad 0<\eta \leqslant 1$

with $q$ given by Eq. (5) and the parameter $\phi$ denoting the rest of terms in the expression given by Wheatcraft and Meerschaert (2008). The fractional flux has two features as Wheatcraft and Meerschaert (2008) explain, which include the scale-invariant property that eliminates the scale effects on parameters. Replacing the rate of change in time with the fractional derivative in Eq. (12) and combining it with Eq. (5) result in

$\phi \frac{\partial^{\beta} h}{\partial t^{\beta}}=\frac{\partial^{\eta}}{\partial x^{\eta}}\left(K h \frac{\partial h}{\partial x}\right)-\frac{\partial^{\eta}}{\partial x^{\eta}}(\omega K h), \quad 0<\eta \leqslant 1$,

Eq. (13) applies to both confined and unconfined aquifers, similar to Eq. (1), with the only difference being the parameters defined for the two different types of aquifers (see Table 1). Eq. (13) is a nonlinear fPDE due to the term $K h$ which has very interesting properties compared to its linear counterpart. In hydrological applications, Eq. (13) can be simplified using the transmissivity, which is a specific term defined differently for unconfined and confined aquifers (see Table 1 ).

Eq. (13) is also applicable to both homogeneous and heterogeneous aquifers depending on whether the hydraulic conductivity, $K$, is a constant or function. In practice, its simplifications are preferred to make their applications easier. In the following cases simplifications are achieved for homogeneous aquifers with $K$ being a constant and for heterogeneous aquifers using the transmissivity or weighted mean for $K$.

It should be noted that the expansion of the term $\frac{\partial^{\eta}}{\partial x^{\eta}}\left(K h \frac{\partial h}{\partial x}\right)$ in Eq. (13) cannot be simply performed using the Leibniz chain rule for integer calculus, and requires a more complex Leibniz chain rule which is a finite series of the product of the integer and fractional derivatives of the two terms, Kh and $\frac{\partial h}{\partial x}$ (Podlubny, 1999, p. 91-97).

\subsubsection{A simplified fractional Boussinesq equation for homogeneous aquifers}

For homogeneous aquifers with $K$ being a constant, Eq. (13) simplifies as

$\phi \frac{\partial^{\beta} h}{\partial t^{\beta}}=K \frac{\partial^{\eta}}{\partial x^{\eta}}\left[h\left(\frac{\partial h}{\partial x}-\omega\right)\right]$

Eq. (14) applies to both confined and unconfined aquifers. With constant $K \mathrm{Eq}$. (14) is still a nonlinear fPDE.

\subsubsection{A simplified fractional Boussinesq equation for heterogeneous confined aquifers}

For heterogeneous confined aquifers, $K$ is a function of the depth, $z$, i.e., $K(z)$, and there are two methods in practice for its determination. One method is the weighted mean hydraulic conductivity for confined aquifers, $\bar{K}_{c}$, recommended to replace the conventional hydraulic conductivity, $K$, for a heterogeneous confined aquifer (Bear, 1972, p. 214) in Eq. (13)

$\bar{K}_{c}=\frac{1}{b} \int_{0}^{b} K(z) d z$

with $b$ being the thickness of the aquifer. Then the weighted transmissivity for confined aquifers, $\bar{T}_{c}$, is

$\bar{T}_{c}=\bar{K}_{c} h$

Another method for the simplification is the use of the mean transmissivity (Bras, 1990, p. 298)

$\bar{T}_{c}=\int_{z_{1}}^{z_{2}} K(z) d z$

where $z_{1}$ and $z_{2}$ are the heights of the aquifer at two points of interest, respectively. Then Eq. (13) for heterogeneous confined aquifers is written as

$\phi \frac{\partial^{\beta} h}{\partial t^{\beta}}=\bar{T}_{c} \frac{\partial^{\lambda} h}{\partial x^{\lambda}}-\omega \bar{K}_{c} \frac{\partial^{\eta} h}{\partial x^{\eta}}$

with $\lambda=\eta+1$. Eq. (18) is a linear fPDE which is different from the nonlinear Eq. (14), and many published reports are available which document analytical solutions of linear fPDEs either through a fractional differential equation (fDE) following the Laplace transform of Eq. (18) (Podlubny, 1999) or its present form (Baleanu et al., 2014).

\subsubsection{A simplified fractional Boussinesq equation for heterogeneous unconfined aquifers}

For heterogeneous unconfined aquifers, the mean transmissivity is given by (Bras, 1990, p. 298)

$\bar{T}_{u}=\int_{z_{1}}^{h} K(z) d z$ 
where the upper integral limit is the free surface of the aquifer rather than a fixed height, and the weighted mean hydraulic conductivity for a heterogeneous unconfined aquifer, $\bar{K}_{u}$, is given by (Bear, 1972, p. 376)

$\bar{K}_{u}=\frac{1}{h} \int_{0}^{h} K(z) d z$

when the lower limit $z_{1}$ is set to zero as the reference datum, which can also apply to Eq. (19).

With Eqs. (19) and (20) for the weighted means, Eq. (13) for heterogeneous unconfined aquifers is now written, with $\lambda=\eta+1$, as

$\phi \frac{\partial^{\beta} h}{\partial t^{\beta}}=\bar{T}_{u} \frac{\partial^{\lambda} h}{\partial x^{\lambda}}-\omega \bar{K}_{u} \frac{\partial^{\eta} h}{\partial x^{\eta}}$

which has an identical structure with Eq. (18) for heterogeneous confined aquifers but the parameters represent different concepts.

The above analyses indicate that for homogeneous aquifers, the same governing equation applies. For heterogeneous aquifers the simplified governing equations (Eqs. (18) and (21)) look identical in structure but the two weighted parameters, $\bar{T}$ and $\bar{K}$, are determined slightly differently with the differences being the upper limit of the integral and the denominator for weighting the integral.

The fractional orders in the above equations, $\beta, \lambda$ and $\eta$, are not necessarily fractions or constants, they can also be functions of time, space and/or $h$ or other parameters. When they are functions, the orders are called variable orders of fractional differentiation (Samko, 1995; Lorenzo and Hartley, 2002). According to Odzijewicz et al. (2013) the concept of variable orders of fractional calculus was initiated by Samko in 1995. In this paper, we only consider aquifers with $\beta, \lambda$ and $\eta$ being fractions.

In the following section, we first discuss "the backwater effect" at a micro-scale which is represented by the backward space fractional derivative, and related issues.

\section{The distributed-order fractional Boussinesq equations, and forward and backward fractional derivatives in the framework of CTRW}

\subsection{The distributed-order fractional Boussinesq equations}

At a large spatial scale, the backwater effect in hydraulics is well known. This phenomenon can be extended to flow at a micro-scale with the backward fractional derivatives in space representing the micro-scale backwater effect. Bochner (1949) introduced the backward and forward fractional linear operators and Saichev and Zaslavsky (1997) described "wandering processes" using the terms "symmetric fractional derivatives" (SFDs) to take into account the backwater effect in particle motion. By definition, the fractional derivatives incorporate those two components - backward and forward fractional derivatives when the symmetric case is considered (Gorenflo and Mainardi, 2001; Umarov and Gorenflo, 2005).

At temporal scales, the flow of fluid through different sizes of pores can be represented by multi-term fractional time derivatives which can account for flow in fractal porous media. The multi-term time fractional derivatives (Jiang et al., 2012, p. 1119) used here take the following form instead of the time fractional derivatives on the left-hand side of the fPDEs,

$P\left(D_{t}\right) h(x, t)=\left(D_{t}^{\beta}+\sum_{i=1}^{s} a_{i} D_{t}^{\beta_{i}}\right) h(x, t)$

where $D_{t}^{\beta}$ and $D_{t}^{\beta_{i}}$ are Caputo fractional derivatives with $0 \leqslant \beta_{s}<\ldots \beta_{1}<\beta \leqslant 1$ as fractional orders of the time-fractional dif- fusion equation or $0 \leqslant \beta_{s}<\ldots \beta_{1}<\beta \leqslant 2$ as fractional orders of the time-fractional wave equation with $i=1,2, \ldots n$. In fact, $0 \leqslant \beta_{s}<\ldots \beta_{1}<\beta \leqslant 2$ is more generic when the multi-term fractional diffusion-wave equation is used. $a_{i}(i=1,2, \ldots s)$ are the coefficients of the time-fractional derivatives which account for the proportional contributions from each fractional time derivative to the sum of the fractional derivatives.

Combining Eq. (22) with Eq. (13) with Eqs. (A3) and (A4) for the SFD using Saichev and Zaslavsky's notation yields

$\phi\left(D_{t}^{\beta}+\sum_{i=1}^{s} a_{i} D_{t}^{\beta_{i}}\right) h(x, t)=K \frac{\partial^{\eta} h}{\partial|x|^{\eta}}\left(h \frac{\partial h}{\partial|x|}\right)-\omega K \frac{\partial^{\eta} h}{\partial|x|^{\eta}}$

for a homogeneous aquifer, and

$\phi\left(D_{t}^{\beta}+\sum_{i=1}^{s} a_{i} D_{t}^{\beta_{i}}\right) h(x, t)=\frac{\partial^{\eta}}{\partial|x|^{\eta}}\left[K(z) h \frac{\partial h}{\partial|x|}\right]-\omega \frac{\partial^{\eta}}{\partial|x|^{\eta}}[K(z) h]$

for a heterogeneous aquifer when $K(z)$ varies with the depth.

Eqs. (23) and (24) are the distributed-order fractional Boussinesq equations (fBEs) incorporating the SFDs. It should be noted here that Eqs. (23) and (24) are extensions to Eqs. (14) and (13), respectively, by including multi-term time fractional derivatives, and $\frac{\partial^{\lambda} h}{\partial|x|^{\lambda}}$ for both the forward and backward fractional components accounting for the forward and backward fractional components if the particle or water parcel motion is regarded as a wandering process (Saichev and Zaslavsky, 1997). Saichev and Zaslavsky (1997), Gorenflo and Mainardi (1998a, 1998b), Benson et al. (2000b), Mainardi et al. (2001) and Umarov and Gorenflo (2005) detailed the SFD and their properties, and Umarov and Gorenflo (2005) show their connections with the fractional derivatives.

The multi-term definition is an ideal way to model flow and particle motion in fractal media which has an unlimited level of micro-structures, a key characteristic of fractal media. Examples of its applications include the widely-used two-term mobileimmobile model of solute transport (Schumer et al., 2003b), water flow in soils (Su, 2012, 2014, 2017), and well hydraulics (Su et al., 2015). The backward fractional derivatives explain "the backwater effect" when water parcels bounce backward during the motion in the CTRW model. This is an extension of the macro-scale backwater effect in hydraulics to explain anomalous motion of particles or water parcels at a pore-scale.

As stated earlier the fractional derivatives incorporate the backward and forward components (Gorenflo and Mainardi, 2001; Umarov and Gorenflo, 2005), then the wandering processes characterised by Eqs. (23) and (24) can be simply written, respectively, as follows by dropping the $|x| \operatorname{sign}$ and using $x$ instead (see also explanations in Appendix A of this paper),

$\phi\left(D_{t}^{\beta}+\sum_{i=1}^{s} a_{i} D_{t}^{\beta_{i}}\right) h(x, t)=K \frac{\partial^{\eta}}{\partial x^{\eta}}\left(h \frac{\partial h}{\partial x}\right)-\omega K \frac{\partial^{\eta} h}{\partial x^{\eta}}$

for a homogeneous aquifer, and

$\phi\left(D_{t}^{\beta}+\sum_{i=1}^{s} a_{i} D_{t}^{\beta_{i}}\right) h(x, t)=\frac{\partial^{\eta}}{\partial x^{\eta}}\left[K(z) h \frac{\partial h}{\partial x}\right]-\omega \frac{\partial^{\eta}}{\partial x^{\eta}}[K(z) h]$

for a heterogeneous aquifer.

The mathematical representation of the physical process is certainly more accurate when more terms are used in the temporal fractional derivatives. However, the challenge with the large number of fractional terms in the formulation is the increasing difficulty of evaluating the parameters $\beta_{i}$. To be consistent with the widely-used mobile-immobile model of water flow and solute transport in porous media (saturated and unsaturated), and its initial success in the extension using the fractional approach (Schumer et al., 2003b; Su et al., 2015), in this paper we only retain 
two terms in $\phi\left(D_{t}^{\beta}+\sum_{i=1}^{s} a_{i} D_{t}^{\beta_{i}}\right) h(x, t)$. With the two-term formulation, we adapt our terminology for groundwater flow (Su et al., $2015)$ to modify Eqs. (25) and (26) as

$b_{2} \frac{\partial^{\beta_{2}} h}{\partial t^{\beta_{2}}}+b_{1} \frac{\partial^{\beta_{1}} h}{\partial t^{\beta_{1}}}=K \frac{\partial^{\eta}}{\partial \boldsymbol{x}^{\eta}}\left(h \frac{\partial h}{\partial x}\right)-\omega K \frac{\partial^{\eta} h}{\partial \boldsymbol{x}^{\eta}}$

for a homogeneous aquifer, and

$b_{2} \frac{\partial^{\beta_{2}} h}{\partial t^{\beta_{2}}}+b_{1} \frac{\partial^{\beta_{1}} h}{\partial t^{\beta_{1}}}=\frac{\partial^{\eta}}{\partial x^{\eta}}\left[K(z) h \frac{\partial h}{\partial x}\right]-\omega \frac{\partial^{\eta}}{\partial x^{\eta}}[K(z) h]$

for a heterogeneous aquifer. In Eqs. (27) and (28), $b_{1}$ and $b_{2}$ are the relative effective porosities in immobile and mobile zones, respectively; $\phi_{i m}, \phi_{m}$ and $\phi$ are the effective porosities in the immobile and mobile zones, and total porosity, respectively, and $\beta_{2}>\beta_{1}$ with $\beta_{2}$ for large pores and $\beta_{1}$ for small pores.

Parallel to Eqs. (18) and (21) that are identical in structure but with two differently weighted parameters, $\bar{T}$ and $\bar{K}$, for confined and unconfined aquifers, respectively, by dropping the subscripts denoting the confined and unconfined aquifers, the two-term $\mathrm{fBE}$ in Eq. (28) for heterogeneous aquifers (confined and unconfined) can be simplified as

$b_{2} \frac{\partial^{\beta_{2}} h}{\partial t^{\beta_{2}}}+b_{1} \frac{\partial^{\beta_{1}} h}{\partial t^{\beta_{1}}}=\bar{T} \frac{\partial^{\lambda} h}{\partial x^{\lambda}}-\omega \bar{K} \frac{\partial^{\eta} h}{\partial x^{\eta}}$

with $\lambda=\eta+1$, and $\bar{T}$ and $\bar{K}$ determined using Eqs. (15)-(17) for confined aquifers and Eqs. (19) and (20) for unconfined aquifers, respectively.

When there is no differentiation between mobile and immobile zones in soils, i.e., $b_{1}=0$ as $b_{1}+b_{2}=1$, and $\beta_{1}=0$, and by writing $\beta_{2}=\beta$, Eqs. (14) and (13) are recovered from Eqs. (27) and (28), respectively.

The applications of symmetric fractional derivatives in modelling solute movement are given by Meerschaert et al. (1999), Benson et al. (2000a) and Schumer et al. (2003a). Zhang et al. (2009) and Benson et al. (2013) provide extensive reviews and analyses of the SFD-based models and have tested some of them using data from the field. The analyses by Zhang et al. (2009) and Benson et al. (2013) are mainly for solute transport which also provide an indirect picture of water flow in porous media as solute movement is coupled to water flow.

Zaslavsky (2002, p. 489-511) analysed the SFD-based fPDE in great detail and showed that the orders of fractional derivatives in the time-space fPDE are also related to the exponents for the two probability density functions in the CTRW theory, and "the critical exponents that characterise the fractal structures of space-time" (Zaslavsky, 2002, p. 507), who also demonstrated the derivation of the SFD-based fPDEs and their applications.

\subsection{Dimensions of the parameters in the fractional Boussinesq equations}

Once the new fPDEs are derived, the next important step is to ensure that the dimensions or units of the parameters in the new fPDEs make sense. For simple fractional models such as the fractional diffusion equation with one-term fractional derivatives in time and space, fractional dimensions can be easily defined. For more complicated fractional models such as Eqs. (27) and (28) and subsequent models in this paper, new definitions are needed for each parameter in the fPDEs so that the dimensions of the fPDE are physically meaningful.

In defining dimensions of the fractional diffusion coefficient in a time-fractional diffusion equation, Kilbas et al. (2006, p. 464) suggested that a new parameter be added to the ordinary diffusion coefficient parameter so that the dimension of the conventional parameter is retained while ensuring a correct dimension in the time-fractional diffusion equation. In their approach, the new fractional diffusion coefficient is given by

$C_{a}=K_{0} \tau^{1-\alpha}$

where

$\alpha$ is the order of the time-fractional diffusion equation;

$K_{0}$ is the classic diffusion coefficient with the dimension $\left[L^{2} / T\right]$, and

$\tau$ is the new time constant parameter which accommodates the new dimensions.

Following Kilbas et al. (2006) we modify the four parameters in Eq. (29) as follows:

$b_{i}=\frac{\phi_{i m}}{\phi} \tau_{1}^{1-\beta_{1}}$

$b_{m}=\frac{\phi_{m}}{\phi} \tau_{2}^{1-\beta_{2}}$

$\bar{T}_{f}=\bar{T} \tau_{t}^{2-\lambda}$

$\bar{K}_{f}=\bar{K} \tau_{k}^{1-\eta}$

where $\tau_{1}, \tau_{2}, \tau_{t}$ and $\tau_{k}$ are parameters for dimension corrections, and $b_{1}, b_{2}, \phi_{i m}, \phi_{m}$ and $\phi$ are defined in Eqs. (27) and (28).

With these modifications to the parameters, Eq. (29) can be updated as

$b_{m} \frac{\partial^{\beta_{2}} h}{\partial t^{\beta_{2}}}+b_{i} \frac{\partial^{\beta_{1}} h}{\partial t^{\beta_{1}}}=\bar{T}_{f} \frac{\partial^{\lambda} h}{\partial x^{\lambda}}-\omega \bar{K}_{f} \frac{\partial^{\eta} h}{\partial x^{\eta}}$

With these modifications, Eq. (35) retains the conventional dimensions if the fractional parameters are absent such as the case for $\beta_{1}=1, \beta_{2}=1, \lambda=2$ and $\eta=1$. The dimensions of Eq. (28) applicable to heterogeneous media are slightly different with those in Eqs. (31)-(34) which should consider the functional form $K(z)$.

Another simple way of correcting the dimensions is to use a relative quantity such as the normalised or reduced hydraulic head (Su et al., 2015) and relative moisture ratio (Su, 2014) which avoid the complication of the dimensions. Due to the possible inconsistency between the dimensions of the fPDE and its solutions in different forms, particularly approximate solutions, it is strongly suggested that the dimensions of the parameters in the final solutions be verified to ensure their physical relevance.

\section{Solutions of fPDEs for hydraulic heads in aquifers subject to the boundary conditions of the first kind}

\subsection{Exact solutions}

Here we present solutions of Eq. (35) for heterogeneous aquifers (confined and unconfined with the two weighted parameters determined for each aquifer). The solutions presented here are subject to the following initial condition (IC) and boundary conditions (BCs),

$$
\begin{aligned}
& h(x, t)=h(x, 0), \quad t=0, \quad 0<x<L \\
& h(x, t)=h(0, t), \quad t \geqslant 0, \quad x=0 \\
& h(x, t)=h(L, t), \quad t \geqslant 0, \quad x=L
\end{aligned}
$$

The hydraulic heads at the start of the reference location, $x=0$ and at the aquifer outlet, $x=L$, are $h(0, t)$ and $h(L, t)$, respectively. For water flow in the aquifer with two levels of pores, the largesmall (or mobile-immobile) porosity model is a reasonable choice. 
In this case, the solutions of Jiang et al. (2012, Eq. (44)) are modified to accommodate this situation

$$
\begin{aligned}
h(x, t)= & \frac{[h(L, t)-h(0, t)] x}{L}+h(0, t)+\sum_{n=1}^{\infty} c_{n 1}(0) u_{0}(t) \\
& \times \sin \left(\frac{n \pi}{L} x\right)
\end{aligned}
$$

where

$c_{n 1}(0)=\frac{2}{L} \int_{0}^{L}\left[\begin{array}{l}h(x, 0)-h(0,0) \\ -\frac{[h(L, 0)-h(0,0)] x}{L}\end{array}\right] \sin \left(\frac{n \pi x}{L}\right) d x$

with $n=1,2, \ldots$. and

$u_{0}(t)=1-k_{n} t^{\beta_{2}} G_{\mu}^{n}(t)$

where $G_{\mu}^{n}(t)$ is the two-variable Mittag-Leffler function (2MLF) given by the following expression (Bhalekar and Daftardar-Gejji, 2013, Eq. (1.9))

$$
\begin{aligned}
G_{\mu}^{n}(t) & =E_{\left(\beta_{2}-\beta_{1}, \beta_{2}\right), 1+\beta_{2}}\left(-\frac{b_{i}}{b_{m}} t^{\beta_{2}-\beta_{1}},-k_{n} t^{\beta_{2}}\right) \\
& =\sum_{j=0}^{\infty} \sum_{i=0}^{j}\left(\begin{array}{l}
j \\
i
\end{array}\right) \frac{\left(-\frac{b_{i}}{b_{m}} t^{\beta_{2}-\beta_{1}}\right)^{i}\left(-k_{n} t^{\beta_{2}}\right)^{j-i}}{\Gamma\left[\left(1+\beta_{2}\right)+\left(\beta_{2}-\beta_{1}\right) i+\beta_{2}(j-i)\right]}
\end{aligned}
$$

where

$$
\left(\begin{array}{l}
j \\
i
\end{array}\right)= \begin{cases}\frac{j !}{i !(j-i) !} & \text { for } 0 \leqslant i \leqslant j \\
0 & \text { for } 0 \leqslant j<i\end{cases}
$$

is the binomial coefficient (Bronshtein and Semendyayev, 1979), and

$k_{n}=\frac{1}{b_{m}}\left[\bar{T}_{f} \lambda_{n}^{\lambda}-\omega \bar{K}_{f} \lambda_{n}^{\eta}\right], \quad n=1,2, \ldots$

with

$\lambda_{n}=\frac{n \pi}{L}, \quad n=1,2, \ldots$

The $2 M L F$ is a special form of the multinomial Mittag-Leffler function (MMLF) (Hadid and Luchko, 1996; Luchko and Gorenflo, 1999; Luchko, 2011). For more details of the MMLF, the reader is referred to Saxena et al. (2011) and Li et al. (2015).

4.2. Particular solutions for hydraulic heads with known values for the IC and $B C S$

The solution presented by Jiang et al. (2012) can be completed by specifying particular values for the IC and BCs, $h(0,0), h(x, 0)$ and $h(L, 0)$. To complete the integral in Eq. (40), let us specify that the initial hydraulic head, $h(x, 0)$, and the values at the two boundaries, $h(0,0)$ and $h(L, 0)$, are constant, then we rewrite Eq. (40) as

$c_{n 1}(0)=\frac{2}{L} \int_{0}^{L}\left(a_{1}+a_{2} x\right) \sin \left(\frac{n \pi}{L} x\right) d x$

with

$a_{1}=h(x, 0)-h(0,0)$

and

$a_{2}=-\frac{[h(L, 0)-h(0,0)]}{L}$

Eq. (46) can be integrated (Gradshteyn and Ryzhik, 1994, p. 227, Eq. (1)), combined with the upper boundary condition which determines the constant of integration to be zero, to yield

$c_{n 1}(0)=\frac{2}{n \pi}\left[\frac{a_{2} L}{n \pi} \sin \left(\frac{n \pi x}{L}\right)-\left(a_{1}+a_{2} x\right) \cos \left(\frac{n \pi x}{L}\right)\right]$
With Eq. (49), the solution given by Eq. (39) can now be finally written

$$
\begin{aligned}
h(x, t)= & h(0, t)+\frac{[h(L, t)-h(0, t)] x}{L} \\
& +\sum_{n=1}^{\infty} \frac{2 \sin \left(\frac{n \pi x}{L}\right)}{n \pi}\left[\frac{a_{2} L \sin \left(\frac{n \pi x}{L}\right)}{n \pi}-\left(a_{1}+a_{2} x\right) \cos \left(\frac{n \pi x}{L}\right)\right] G_{n}(t)
\end{aligned}
$$

with

$G_{n}(t)=\left[1-k_{n} t^{\beta_{2}} E_{\left(\beta_{1}, \beta_{2}\right), 1+\beta_{2}}\left(-\frac{b_{i}}{b_{m}} t^{\beta_{1}},-k_{n} t^{\beta_{2}}\right)\right]$

It is clear from Eq. (50) that the hydraulic head at the lower boundary at $x=L$ is given by $h(x, t)=h(L, t)$. For $t=0$, Eq. (50) yields the initial water level in different locations as follows

$$
\begin{aligned}
h(x, 0)= & h(0,0)+\frac{[h(L, t)-h(0,0)] x}{L} \\
& +\sum_{n=1}^{\infty} \frac{2}{n \pi} \sin \left(\frac{n \pi x}{L}\right)\left[\frac{a_{2} L}{n \pi} \sin \left(\frac{n \pi x}{L}\right)-\left(a_{1}+a_{2} x\right) \cos \left(\frac{n \pi x}{L}\right)\right]
\end{aligned}
$$

\subsection{Approximate solutions of hydraulic heads in an aquifer}

For practical application, the solutions in Eq. (50) can be approximated by retaining only limited terms. One approximation is made for $n=1$, which yields

$$
\begin{aligned}
h(x, t)= & h(0, t)+\frac{[h(L, t)-h(0, t)] x}{L} \\
& +\frac{1}{\pi}\left\{\frac{a_{2} L}{\pi}\left[1-\cos \left(\frac{2 \pi x}{L}\right)\right]-\left(a_{1}+a_{2} x\right) \sin \left(\frac{2 \pi x}{L}\right)\right\} G_{1}(t)
\end{aligned}
$$

where $G_{1}(t)$ is given by Eq. (51) with $n=1$ and

$k_{1}=\frac{1}{b_{m}}\left[\bar{T}_{f} \lambda_{1}^{\lambda}-\omega \bar{K}_{f} \lambda_{1}^{\eta}\right]$

In the above solutions, the 2 MLF can also be approximated by retaining limited terms. Retaining only two leading terms in the 2MLF in Eq. (42) with $j=0,1$ and $i=0,1$ results in

$$
\begin{aligned}
E_{\left(\beta_{2}-\beta_{1}, \beta_{2}\right), 1+\beta_{2}}\left(-\frac{b_{i}}{b_{m}} t^{\beta_{2}-\beta_{1}},-k_{1} t^{\beta_{2}}\right) \\
=\frac{1}{\Gamma\left[1+\beta_{2}\right]}+\frac{b_{i} t^{-\beta_{1}}}{k_{1} b_{m} \Gamma\left[1+\beta_{2}-\beta_{1}\right]}-\frac{k_{1} t^{\beta_{2}}}{\Gamma\left[1+2 \beta_{2}\right]} \\
\quad-\frac{b_{i} t^{\beta_{2}-\beta_{1}}}{b_{m} \Gamma\left[1+2 \beta_{2}-\beta_{1}\right]}
\end{aligned}
$$

then the approximate solution of Eq. (53) becomes

$h(x, t)=h(0, t)+a_{0} x+\left\{\begin{array}{l}\frac{a_{2} L}{\pi}\left[1-\cos \left(\frac{2 \pi x}{L}\right)\right] \\ -\left(a_{1}+a_{2} x\right) \sin \left(\frac{2 \pi x}{L}\right)\end{array}\right\} \frac{\left(1-F_{t}\right)}{\pi}$

where

$a_{0}=\frac{[h(L, t)-h(0, t)]}{L}$

and

$F_{t}=k_{1} t^{\beta_{2}}\left(\begin{array}{l}\frac{1}{\Gamma\left[1+\beta_{2}\right]}+\frac{b_{i} t^{-\beta_{1}}}{k_{1} b_{m} \Gamma\left[1+\beta_{2}-\beta_{1}\right]} \\ -\frac{k_{1} t^{\beta_{2}}}{\Gamma\left[1+2 \beta_{2}\right]}-\frac{b_{i} t^{\beta_{2}-\beta_{1}}}{b_{m} \Gamma\left[1+2 \beta_{2}-\beta_{1}\right]}\end{array}\right)$

At $x=0$, Eq. (56) becomes $h(0, t)$, and at $x=L$, it becomes $h(x, t)=h(L, t)$ for $\cos \left(\frac{2 \pi x}{L}\right)=1$ and $\sin \left(\frac{2 \pi x}{L}\right)=0$. 
Eq. (56) can be used to determine the dynamics of the aquifer given the initial and boundary values of $h(x, t)$, namely, $a_{0}, a_{1}$ and $a_{2}$ in Eqs. (57), (47) and (48), respectively.

$F_{t}$ in Eq. (58) should be dimensionless so that the dimension of the hydraulic head $h(x, t)$ in Eq. (56) is $[L]$, which requires that $k_{1}$ has the dimension of $\left[T^{-\beta_{2}}\right]$ and also $\frac{b_{i}}{b_{m}}$ to have dimensions of $T^{\beta_{1}-\beta_{2}}$ which changes the definitions of $b_{i}$ and $b_{m}$ to embrace dimensions as stated earlier. Due to the infinite terms in the original multi-term solutions, the dimensions of other parameters such as $k_{2}, k_{3}$ and $k_{i}$ also change.

\section{Equations of groundwater discharge from aquifers}

The above solutions are applicable to both confined and unconfined aquifers with the weighted transmissivity and hydraulic conductivity being defined and determined for each aquifer. Here we consider the application of the solutions as an example.

Here we use the simplified solution in Eq. (56) and the discharge given by Eq. (5) to derive recession curves. Differentiating Eq. (56) with respect to $x$ and combining the result with Eq. (5) yields the discharge of the aquifer per unit width, $q$,

$q=\bar{K}_{f} h(L, t)\left[\omega-\xi_{t}+2 \xi_{0}\left(1-F_{t}\right)\right]$

where

$\xi_{t}=\frac{[h(L, t)-h(0, t)]}{L}$

is the average gradient of the aquifer at any time with $F_{t}$ in Eq. (58), and

$\xi_{0}=\frac{[h(L, 0)-h(0,0)]}{L}=-a_{2}$

is the average gradient of the aquifer at the initial time.

The unit discharge, $q$, has a unit of $L^{2} / T$ such as $\mathrm{m}^{2} / \mathrm{h}$. The total discharge from an aquifer is

$Q=q w$

where $w$ is the width of the aquifer.

In the following situations, Eq. (59) can be further simplified.

\subsection{Case 1: The aquifer on a horizontal base}

For an aquifer on a horizontal base, $\omega=0$, Eq. (59) becomes

$q=\bar{K}_{f} h(L, t)\left[2 \xi_{0}\left(1-F_{t}\right)-\xi_{t}\right]$

If the two gradients in Eqs. (60) and (61) are equal, i.e., $\xi_{0}=\xi_{t}$, or approximately equal, $\xi_{0} \approx \xi_{t}$, then we can assign both as $\xi$, and Eq. (63) becomes

$q=\bar{K}_{f} h(L, t) \xi\left(1-2 F_{t}\right)$

In the above expressions, $F_{t}$ is given by Eq. (58).

\subsection{Case 2: The initial discharge}

In Eq. (59), for $t=0, F_{t}=0$, one arrives at

$q=\bar{K}_{f} h(L, t)\left(\omega+2 \xi_{0}-\xi_{t}\right)$

which means that the initial discharge of the aquifer from the lower end depends on the slope gradient, $\omega$, and the gradients of the aquifer.

For an aquifer on a horizontal base, i.e., $\omega=0$, Eq. (65) becomes

$q=\bar{K}_{f} h(L, t)\left(2 \xi_{0}-\xi_{t}\right)$

Also if $\xi_{0}=\xi_{t}$ or $\xi_{0} \approx \xi_{t}$, and we assign both as $\xi$, Eq. (66) becomes
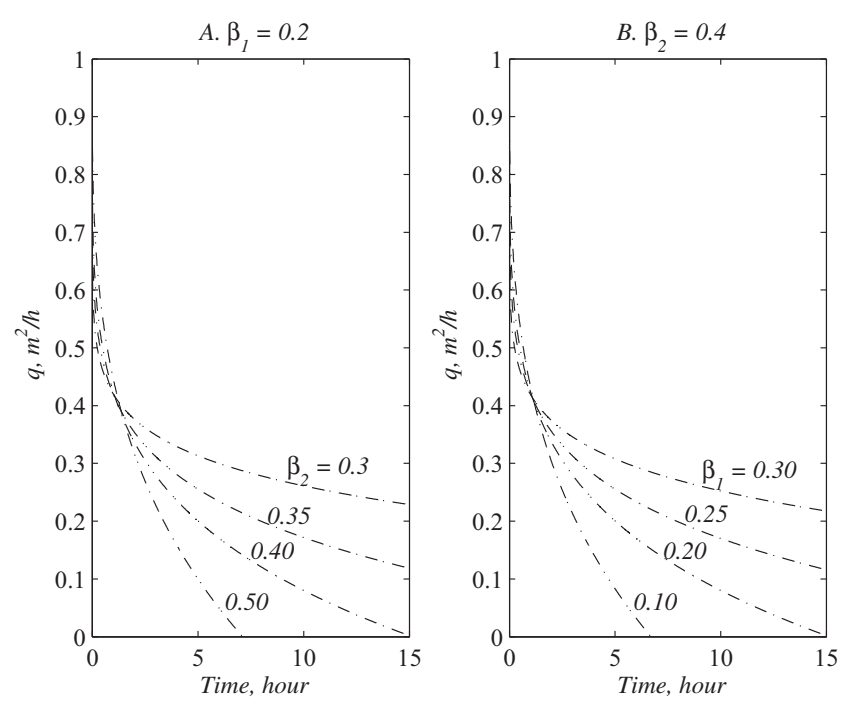

Fig. 1. The effects of the orders of the fractional derivatives, $\beta_{2}$ and $\beta_{1}$, on the recession curves illustration by Eq. (64). (A) Fixed $\beta_{1}$ with variable $\beta_{2}$ indicating the facilitating role of $\beta_{2}$ in large pores. (B) Fixed $\beta_{2}$ with variable $\beta_{1}$ indicating the trapping role of $\beta_{1}$ in small pores.

$q=\bar{K}_{f} h(L, t) \xi$

which is a simple statement that the discharge from the end of the aquifer is a product of the hydraulic conductivity, the hydraulic head, and the hydraulic gradient, and is precisely a result of the definition of the discharge in Eq. (5). The above simplifications are also a verification of all the derivations based on fractional calculus.

As an example, we use Eq. (64) to demonstrate its applications when the aquifer is on a horizontal base with $\omega=0$. A graphical illustration of Eq. (64) is shown in Fig. 1. The parameters used in Fig. 1 are hypothetical for illustration only, which are $\bar{K}_{f} h(L, t) \xi_{0}=1.0, b_{i}=0.2, b_{m}=0.8$, and $k_{1}=0.01$.

The illustration in Fig. 1 implies that the increase in the large porosity represented by an increased $b_{m}$ facilitates the groundwater drainage while the increase in the small porosity slows down the drainage due to the decreasing large pores. This fact is intuitive and realistic.

\section{Conclusion and discussions}

In this paper, a set of two-term distributed-order time-space symmetrical fractional Boussinesq equations (fBEs) for groundwater flow have been presented, which are applicable to both confined and unconfined aquifers. A simplified $\mathrm{fBE}$ is presented and solutions given by modifying generic solutions given by Jiang et al. (2012). The fBEs are formulated based on the symmetrical fractional derivatives (SFD) in space for both diffusion and advection which explain random wandering processes (Saichev and Zaslavsky, 1997).

The SFD consists of the forward fractional derivative (FFD) and the backward fractional derivative (BFD). The FFD is understood as flow in the direction of the diffusion and advection consistent with the traditional definitions. The BFD, however, can be interpreted as the micro-scale backwater effect. The continuous-time random walk (CTRW) theory with symmetrical fractional derivatives provides an alternative for deriving the fundamental equation governing the flow without resorting to the traditional method of the conservation of mass.

In the $\mathrm{fBE}$, two-term fractional derivatives in time are used to model flow in large and small pores to be consistent with the well-known mobile-immobile (or large-small pore) model. By 
modifying the solutions in Jiang et al. (2012), analytical solutions of the $\mathrm{fBE}$ are presented with known heads at both boundaries, and the solutions are presented in completed algebraic forms with simplified solutions given. Based on the solutions, a set of simplified formulae for groundwater discharge for different cases are presented. These formulae are very simple and easy to use for estimating groundwater recession and for evaluating the parameters in the fBE such as the parameters in $F_{t}$. Some of the formulae for groundwater recession curves have also been illustrated.

As discussed in Eq. (A15) and (A16) in Appendix A, the sign for SFDs $\frac{\partial^{\lambda} h}{\partial \mid x^{\lambda}}$ and $D_{0}^{\lambda} h$ are identical, then $D_{0}^{\lambda} h=\frac{\partial^{\lambda} h}{\partial x^{\lambda}}$ is used throughout the text as the synonyms of the symmetric fractional derivatives.

For the fBEs presented for a heterogeneous aquifer, either a weighted average transmissivity, $\bar{T}$, or the nonlinear fBEs can be used. While the average transmissivity is widely used, we draw particular attention to the fact that, for a specific form of the hydraulic conductivity, $K(z)=\frac{1}{\lambda} \Gamma(1+\lambda) z^{1-\lambda}$, Voller (2011, p. 260261 ) shows that the integer PDE of the form $\frac{\partial}{\partial z}\left(K(z) \frac{\partial h}{\partial z}\right)=0$ yields an identical result for the wetting front derived using a fractional model under the same boundary conditions. This fact implies that the fPDE itself is equivalent to the classic PDE with a spacedependent conductivity. In this regard, the use of an average transmissivity is not necessary because the fPDE itself accounts for spatial variability or the non-local effect if the hydraulic conductivity takes the form of $K(z)=\frac{1}{\lambda} \Gamma[1+\lambda] z^{1-\lambda}$, where $\Gamma[1+\lambda]$ is the gamma function.

For physical meanings of the fractional integration and fractional derivatives, the reader is referred to Podlubny (2002) and Heymans and Podlubny (2006). An important property of the explanations by Podlubny (2002) is that fractional integration, which defines the fractional derivatives, is history-dependent which is ideally represented in the CTRW concept.

In the literature, anomalous diffusion is extensively investigated in physics and mathematics, and solute transport in aquifers have also been extensively studied, but the specialisation of fPDE models for fluid flow in aquifers has only been attempted recently (see Su et al., 2015 for a brief review). A set of fBEs in this paper is a further extension to our earlier investigation which shows that the two-term distributed-order fPDE is capable of capturing water flow in both large and small pores which has been qualitatively interpreted without a parameter to quantify it (Bouwer, 1989). To this end the methods presented earlier (Su et al., 2015) and this paper reinforce our understanding of water flow in aquifers at a more detailed level. We expect that more solutions of the fBEs subject to different conditions will provide new insights into how water flows in fractal media.

The fBEs presented here are applicable to both confined and unconfined aquifers. We should note that many published reports on the application of fractional models in solute transport in aquifers do not consider the distinction between confined and unconfined aquifers.

\section{Acknowledgements}

The author wishes to acknowledge the comments made by anonymous reviewers which helped improve the manuscript. The research presented here was partly supported by the "Distinguished Expert of Ningxia" programme.

\section{Appendix A. Appendix A}

In the CTRW theory, particles (or water parcels) can move in both forward and backward directions during their motion. Their movements in the two directions can be represented by backward and forward space fractional derivatives (Saichev and Zaslavsky, 1997).

For homogeneous and heterogeneous aquifers, Eqs. (27) and (28) are, respectively, written with the fractional differential operator, $\hat{L}(x)$,

$b_{2} \frac{\partial^{\beta_{2}} h}{\partial t^{\beta_{2}}}+b_{1} \frac{\partial^{\beta_{1}} h}{\partial t^{\beta_{1}}}=\hat{L}_{h}(x) h$

and

$b_{2} \frac{\partial^{\beta_{2}} h}{\partial t^{\beta_{2}}}+b_{1} \frac{\partial^{\beta_{1}} h}{\partial t^{\beta_{1}}}=\hat{L}_{i}(x) h$

where

$\hat{L}_{h}(x) h=K \frac{\partial^{\eta}}{\partial|x|^{\eta}}\left(h \frac{\partial h}{\partial|x|}\right)-\omega K \frac{\partial^{\eta} h}{\partial|x|^{\eta}}$

is for a homogeneous aquifer, and

$\hat{L}_{i}(x) h=\frac{\partial^{\eta}}{\partial|x|^{\eta}}\left[K(z) h \frac{\partial h}{\partial|x|}\right]-\omega \frac{\partial^{\eta}}{\partial|x|^{\eta}}[K(z) h]$

is for a heterogeneous aquifer with $\frac{\partial^{\lambda} h}{\partial|x|^{\lambda}}$ and $\frac{\partial^{\eta} h}{\partial|x|^{\eta}}$ being Riesz space fractional derivatives (RSFD). For a homogeneous aquifer which has a transmissivity, $T$, the RSFD is defined as (Saichev and Zaslavsky, 1997),

$$
\begin{aligned}
\hat{L}_{h}(x) h & =D^{+} \frac{\partial^{\lambda} h}{\partial x^{\lambda}}+D^{-} \frac{\partial^{\lambda} h}{\partial(-x)^{\lambda}} \\
& -V^{+} \frac{\partial^{\eta} h}{\partial x^{\eta}}-V^{-} \frac{\partial^{\eta} h}{\partial(-x)^{\eta}}
\end{aligned}
$$

$D^{+}=\frac{T^{+}}{\phi}$

$D^{-}=\frac{T^{-}}{\phi}$

$V^{+}=-\frac{\omega K^{+}}{\phi}$

$V^{-}=-\frac{\omega K^{-}}{\phi}$

For an inhomogeneous aquifer which has a transmissivity, $\bar{T}$, the above formulations apply except for $T$ to be replaced by $\bar{T}$.

The forward motion is represented by the forward fractional derivatives, $D^{+} \frac{\partial^{\lambda} h}{\partial x^{i}}$ and $V^{+} \frac{\partial^{\eta} h}{\partial x^{\eta}}$, while the backward motion by the backward fractional derivative, $D^{-} \frac{\partial^{\lambda} h}{\partial(-x)^{\lambda}}$ and $V^{-} \frac{\partial^{\eta} h}{\partial(-x)^{\eta}}$. These terms with different parameters, $D^{+}, D^{-}, V^{+}$and $V^{-}$manifest the flow mechanics and anistrophic properties of the media.

For a simplified situation when the media is isotropic where $D^{+}=D^{-}=D^{\lambda}$, and $V^{+}=V^{-}=V^{\eta}$, Saichev and Zaslavsky (1997) defined the fractional diffusion coefficient, $D$,

$D=-2 D^{\lambda} \cos \left(\frac{\pi \lambda}{2}\right)$

which can be extended to the fractional convection velocity, $V$,

$V=-2 V^{\eta} \cos \left(\frac{\pi \eta}{2}\right)$

then the symmetric fractional derivatives (SFDs) are defined as

$\frac{\partial^{\lambda} h}{\partial|x|^{\lambda}}=-\frac{1}{2 \cos \left(\frac{\pi \lambda}{2}\right)}\left(\frac{\partial^{\lambda} h}{\partial x^{\lambda}}+\frac{\partial^{\lambda} h}{\partial|-x|^{\lambda}}\right)$ 
and

$\frac{\partial^{\eta} h}{\partial|x|^{\eta}}=-\frac{1}{2 \cos \left(\frac{\pi \eta}{2}\right)}\left(\frac{\partial^{\eta} h}{\partial x^{\eta}}+\frac{\partial^{\eta} h}{\partial|-x|^{\eta}}\right)$

Further discussions of the symmetric fractional calculus are also provided by Saichev and Zaslavsky (1997), Gorenflo and Mainardi (1998a, 1998b), Mainardi et al. (2001) and Umarov and Gorenflo (2005). Two of important properties of SFDs are that $\frac{\partial^{\lambda} h}{\partial|x|^{\lambda}} \neq 0$ is defined for $x>0$ and $\frac{\partial^{\lambda} h}{\partial|x|^{\lambda}}=0$ for $x<0$, and on the contrary, $\frac{\partial^{2} h}{\partial|-x|^{\lambda}} \neq 0$ is defined for $x<0$ and $\frac{\partial^{\lambda} h}{\partial|-x|^{\lambda}}=0$ for $x>0$ (Saichev and Zaslavsky, 1997, p. 762). To be consistent with the notations used by Saichev and Zaslavsky (1997, p. 762), $\frac{\partial h}{\partial|x|}$ is also retained when the derivative is an integer.

Umarov and Gorenflo (2005, p. 79) show that the pseudodifferential operator for fractional derivatives are identical to the fractional power of the Laplace operator,

$D_{0}^{\lambda}=-(-\Delta)^{\lambda / 2}$

For one-dimensional fractional derivatives of the known function $h$, the operator in Eq. (A14) is written as

$D_{0}^{\lambda} h=-\left(-\frac{\partial^{2}}{\partial x^{2}}\right)^{\lambda / 2} h$

For a finite domain $[0, L ; 0, T]$ with the homogeneous boundary conditions $h(0, t)=h(L, t)=0$, Jiang et al. (2012, Eq. (9)) show that the following equality holds in one dimension

$-\left(-\frac{\partial^{2}}{\partial x^{2}}\right)^{\lambda / 2} h=-c_{\lambda}\left({ }_{0} D_{x}^{\lambda} h+{ }_{x} D_{L}^{\lambda} h\right)=\frac{\partial^{\lambda} h}{\partial|x|^{\lambda}}$

where ${ }_{0} D_{x}^{\lambda} h$ and ${ }_{x} D_{L}^{\lambda} h$ are the left-sided and right-sided fractional derivatives, respectively, and

$c_{\lambda}=\frac{1}{2 \cos \left(\frac{\lambda \pi}{2}\right)}, \quad \lambda \neq 1$

Comparing Eq. (A15) and (A16) shows that the sign for SFDs $\frac{\partial^{\lambda} h}{\partial|x|^{\lambda}}$ and $D_{0}^{\lambda} h$ are identical, then $D_{0}^{\lambda} h=\frac{\partial^{\lambda} h}{\partial x^{\lambda}}$ and $D_{0}^{\eta} h=\frac{\partial^{\eta} h}{\partial x^{\eta}}$ is used throughout the text as the synonyms of the symmetric fractional derivatives.

In Eqs. (A10) and (A11), the dimensions of $D$ and $V$ depend on the value of $\lambda$ and $\eta$. Their dimensions in exact solutions should remain the same as in the main model equations. However, due to approximations, their dimensions could change depending on how the approximations are made.

\section{References}

Abdellaoui, B., Peral, I., Walias, 2015. Some existence and regularity results for porous media and fast diffusion equations with a gradient term. Trans. Am. Math. Soc. 367, 4757-4791.

Alkahtani, B.S.T., Atangana, A 2016. Controlling the wave movement on the surface of shallow water with the Caputo-Fabrizio derivative with fractional order. Chaos, Solitons Fractals 89, 539-546.

Atangana, A. 2014. Drawdown in prolate spheroidal-spherical coordinates obtained via Green's function and perturbation methods. Commun. Nonlinear Sci. Numer. Simulat. 19 (5), 1259-1269.

Atangana, A., 2016. Derivative with two fractional orders: a new avenue of investigation toward revolution in fractional calculus. Eur. Phys. J. Plus 131 (10), 373.

Atangana, A., Alkahtani, B.S.T., 2016. New model of groundwater flowing within a confine aquifer: application of Caputo-Fabrizio derivative. Arab. J. Geosci. 9 (1), $1-6$.

Atangana, A., Baleanu, D., 2016. New fractional derivatives with non-local and nonsingular kernel: theory and application to heat transfer model. Therm. Sci. 20 (2), 763-769. arXiv:1602.03408.

Atangana, A., Bildik, N., 2013. The use of fractional order derivative to predict the groundwater flow. Math. Prob. Eng. 2013 (543026), 1-9.
Atangana, A., Vermeulen, P.D., 2014. Analytical solutions of a space-time fractional derivative of groundwater flow equation. Abstr. Appl. Anal. 2014 (381753), 111.

Barenblatt, G.I., Bertsch, M., Chertock, A.E., Prostokishin, V.M., 2000. Self-similar intermediate asymptotics for a degenerate parabolic filtration-absorption equation. Proc. Nat. Acad. Sci. 97 (18), 9844-9848.

Bear, J., 1972. Dynamics of Fluids in Porous Media. Dover, NY.

Baleanu, D., Wu, G.C., Duan, J.S., 2014. Some analytical techniques in fractional calculus: realities and challenges. In: Machado, J.A.T. et al. (Eds.), Discontinuity and Complexity in Nonlinear Physical Systems, Nonlinear Systems and Complexity, 6. Springer Internl. Publ., Switzerland, pp. 35-62. Chapter 3.

Benson, D.A., 1998. The fractional advection-dispersion equation: development and application PhD dissertation. Univ. Nevada, Reno.

Benson, D.A., Meerschaert, M.M., Revielle, J., 2013. Fractional calculus in hydrologic modelling: a numerical perspective. Adv. Water Resour. 51, 479-497.

Benson, D.A. Wheatcraft, S.W., Meerschaert, M.M., 2000a. Application of a fractional advection-dispersion equation. Water Resour. Res. 36 (6), 1403-1412.

Benson, D.A., Wheatcraft, S.W., Meerschaert, M.M., 2000b. The fractional order governing equation of Lévy motion. Water Resour. Res. 36 (6), 1413-1423.

Berkowitz, B., Scher, H., 1995. On characterization of anomalous dispersion in porous and fractured media. Water Resour. Res. 31 (6), 1461-1466.

Berkowitz, B., Klafter, J., Metzler, R., Scher, H., 2002. Physical pictures of transport in heterogeneous media: advection-dispersion, random-walk, and fractional derivative formulations. Water Resour. Res. 38 (10), 1191. http://dx.doi.org/ 10.1029/2001WR001030.

Berkowitz, B., Cortis, A., Dentz, M., Scher, H., 2006. Modeling non-Fickian transport in geological formations. Rev. Geophys. 44. 2005RG000178, RG2003/2006.

Bhalekar, S., Daftardar-Gejji, V., 2013. Corrigendum. Appl. Math. Comput. 219, 8413-8415.

Bochner, S., 1949. Diffusion equation and stochastics processes. Proc. Nat. Acad. Sci. 35, 368-370.

Boussinesq, M.J., 1904. Recherches théoriques sur l'écoulement des nappes d'eau infiltrées dans le sol et sur débit de sources. J. Math. Pure Appl. 10, 5-78.

Bouwer, H., 1989. The Bouwer and Rice slug test - an update. Groundwater 27 (3), 304-309.

Bras, R.L., 1990. Hydrology. Addison-Wesley, Reading, England.

Bronshtein, I.N., Semendyayev, K.A., 1979. Handbook of Mathematics. Verlag Harri Deutsch, Van Nostrand Reinhold Co., NY.

Cox, D.R., 1967. Renewal Theory. Methuen, London.

Crofton, M.W., 1865. Question 1773. Math. Quest. Solut. Educ. Times 4, 71-72.

Darcy, H., 1856. Les Fontaines Publiques De La Ville De Dijon. Dalmont, Paris.

Dentz, M., Berkowitz, B., 2003. Transport behavior of a passive solute in continuous time random walks and multirate mass transfer. Water Resour. Res. 39 (5), 1111. http://dx.doi.org/10.1029/2001WR001163.

Dentz, M., Kang, P.K., Le Borgne, T., 2015. Continuous time random walks for nonlocal radial solute transport. Adv. Water Resour. 82, 16-26.

Diida, J.D., Area, I., Atangana, A., 2016. New numerical scheme of Atangana-Baleanu fractional integral: an application to groundwater flow within leaky aquifer. arXiv:1610.08681

Gorenflo, R., Mainardi, F., 1998a. Fractional calculus and stable probability distributions. Arch. Mech. 59 (3), 377-388.

Gorenflo, R., Mainardi, F., 1998b. Random walk models for space-fractional diffusion processes. Fract. Calc. Appl. Anal. 1, 167-191.

Gorenflo, R., Mainardi, F., 2001. Random Walk Models Approximating Symmetric Space-Fractional Diffusion Processes. Series Operator Theory: Advances and Applications. Problems and Methods in Mathematical Physics, vol. 121. Birkhäuser, Verlag Basel, Switzerland, pp. 120-145.

Gorenflo, R., Mainardi, F., 2005. Simply and multiply scaled diffusion limits for continuous time random walk. J. Phys: Conf. Ser. 7, 1-16.

Gorenflo, R., Mainardi, F., 2009. Some recent advances in theory and simulation of fractional diffusion processes. J. Comput. Appl. Math. 229, 400-415.

Gorenflo, R., Mainardi, F., 2012. Parametric subordination in fractional diffusion processes. In: Fractional Dynamics. In: Lim, S.C., Klafter, J., Metzler, R. (Eds.), . Recent Advances, vol. 1. World Scientific, Singapore, pp. 227-261.

Gorenflo, R., Mainardi, F., Vivoli, A., 2007. Continuous-time random walk and parametric subordination in fractional diffusion. Chaos, Solitons Fractals 34, $87-103$.

Gradshteyn, I.S., Ryzhik, I.M., 1994. Table of Integrals, Series and Products. Acad. Press, San Diego.

Hadid, S.B., Luchko, Yu., 1996. An operational method for solving fractional differential equations of an arbitrary order. Panamer. Math. J. 6 (1), 57-73.

He, J.H., 1998. Approximate analytical solution for seepage flow with fractional derivatives in porous media. Comput. Methods Appl. Mech. Eng. 167, 57-68.

Heymans, N., Podlubny, I., 2006. Physical interpretation of initial conditions for fractional differential equations with Riemann-Liouville fractional derivatives. Rheol. Acta 45, 765-771.

Jiang, H., Liu, F., Turner, I., Burrage, 2012. Analytical solutions for the multi-term time-space Caputo-Riesz fractional advection-diffusion equations on a finite domain. J. Math. Anal. Appl. 389, 1117-1127.

Kilbas, A.A., Srivastava, H.M., Trujillo, J.J., 2006. Theory and Applications of Fractional Differential Equations. Elsevier, Amsterdam.

Li, Z., Liu, Y., Yamamoto, M., 2015. Initial-boundary value problems for multi-term time-fractional diffusion equations with positive constant coefficients. Appl Math. Comput. 257, 381-397.

Lorenzo, C.F., Hartley, T.T., 2002. Variable order and distributed order fractional operators. Nonlinear Dyn. 29, 57-98. 
Luchko, Yu., 2011. Initial-boundary-value problems for the generalized multi-term time-fractional diffusion equation. J. Math. Anal. Appl. 374, 538-548.

Luchko, Yu., Gorenflo, R., 1999. An operational method for solving fractional differential equations with the Caputo derivatives. Acta Math. Vietn. 24 (2), 207-233.

Mainardi, F., Luchko, Y., Pagnini, G., 2001. The fundamental solution of the spacetime fractional diffusion equation. Fract. Calc. Appl. Anal. 4 (2), 153-192.

Meerschaert, M.M., 2012. In: Metzler, R., Lim, S.C., Klafter, J. (Eds.), Fractional Calculus, Anomalous Diffusion and Probability. World Scientific, Singapore, pp. 265-284.

Meerschaert, M.M., Benson, D.A., Bäumer, B., 1999. Multidimensional advection and fractional dispersion. Phys. Rev. E 59 (5), 5026-5028.

Meerschaert, M.M., Benson, D.A., Scheffler, H.-P., Becker-Kern, P., 2002. Governing equations and solutions of anomalous random walk limits. Phys. Rev. E 66, 060102(R).

Mehdinejadiani, B., Jafari, H., Baleanu, D., 2013. Derivation of a fractional Boussinesq equation for modelling unconfined groundwater flow. Eur. Phys. J. Spec. Top. 222, 1805-1812.

Montroll, E.W., Weiss, G.H., 1965. Random walks on lattices, II. J. Math. Phys. 6 , $167-181$.

Odzijewicz, T., Malinowska, A.B., Torres, D.F.M., 2013. Fractional variational calculus of variable order. In: Almeida, A., Castro, L., Speck, F.-O. (Eds.), Advances in Harmonic Analysis and Operator Theory, The Stefan Samko Anniversary, pp. 291-301.

Ortigueira, M.D., Trujillo, J.J., 2012. A unified approach to fractional derivatives. Commun. Nonlinear Sci. Numer. Simul. 17, 5151-5157.

Pearson, K., 1905. The problem of the random walk. Nature 72 (1867), 294.

Podlubny, I., 1999. Fractional Differential Equations. Acad. Press, San Diego, California.

Podlubny, I., 2002. Geometric and physical interpretation of fractional integration and fractional differentiation. Fract. Calc. Appl. Anal. 5 (4), 367-386.

Saffman, P.G., 1959. A theory of dispersion in a porous medium. J. Fluid Mech. 6 (3), 321-349.

Saichev, A., Zaslavsky, D., 1997. Fractional kinetic equations: solutions and applications. Chaos: Interdisc. J. Nonlinear Sci. 7 (4), 753-764.

Samko, S.G., 1995. Fractional integration and differentiation of variable order. Anal. Math. 21, 213-236.

Saxena, R.K., Kalla, S.L., Saxena, R., 2011. Multivariate analogue of generalized Mittag-Leffler function. Integral Transf. Sp. Funct. 22 (7), 533-548.
Schumer, R., Benson, D.A., Meerschaert, M.M., Baeumer, B., 2003a. Multiscaling fractional advection-dispersion equations and their solutions. Water Resour. Res. 39 (1). 1022, 1-11.

Schumer, R., Benson, D.A., Meerschaert, M.M., Baeumer, B., 2003b. Fractal mobileimmobile solute transport. Water Resour. Res. 39 (10). 1296, 1-12.

Su, N., 2012. Distributed-order infiltration, absorption and water exchange in mobile and immobile zones of swelling soils. J. Hydrol. 468, 1-10.

Su, N., 2014. Mass-time and space-time fractional partial differential equations of water movement in soils: theoretical framework and application to infiltration. J. Hydrol. 519, 1792-1803.

Su, N., Nelson, P.N., Connor, S., 2015. The distributed-order fractional diffusionwave equation of groundwater flow: theory and application to pumping and slug tests. J. Hydrol. 529, 1262-1273.

Su, N., 2017. Exact and approximate solutions of fractional partial differential equations for water movement in soils. Hydrol. 4 (1). http://dx.doi.org/10.3390/ hydrology4010008. Article 8.

Telyakovskiy, A.S., Kurita, S., Allen, M.B., 2016. Polynomial-based approximate solutions to the Boussinesq equation near a well. Adv. Water Resour. 96, 68-73.

Uchaikin, V.V., Saenko, V.V., 2003. Stochastic solution to partial differential equations of fractional orders. Siber. J. Numer. Math. 6 (2), 197-203.

Umarov, S., Gorenflo, R., 2005. On multi-dimensional random walk models approximating symmetric space-time-fractional diffusion processes. Fract. Calc. Appl. Anal. 8 (1), 73-88.

Voller, V.R., 2011. On a fractional derivative form of the Green-Ampt infiltration model. Adv. Water Resour. 34 (2), 257-262.

Werner, P.W., 1957. Some problems in non-artesian ground-water flow. Trans. Am. Geophys. Union 38 (4), 511-518.

Wheatcraft, S.W., Meerschaert, M.M., 2008. Fractional conservation of mass. Adv. Water Resour. 31, 1377-1381.

Zaslavsky, G.M., 2002. Chaos, fractional kinetics, and anomalous transport. Phys. Rep. 371, 461-580.

Zhang, Y., Benson, D.A., Reeves, D.M., 2009. Time and space nonlocalities underlying fractional-derivative models: distinction and literature review of field applications. Adv. Water Resour. 32, 561-581.

Zhuang, P., Liu, F., Turner, I., Gu, Y.T., 2014. Finite volume and finite element methods for solving a one-dimensional space-fractional Boussinesq equation. Appl. Math. Model. 38, 3860-3870. 